\title{
Paliación del cáncer de colon: estoma vs. prótesis
}

\author{
Jony Cerna-Cardona* \\ Unidad de Endoscopia, Hospital Juárez de México, Ciudad de México, México
}

\begin{abstract}
Resumen
Un número considerable de pacientes con cáncer de colon (CC) se manifiestan clínicamente con datos de obstrucción colónica (8-13\%). Existen dos terapias puentes a la cirugía: colocación de prótesis metálicas autoexpandibles (PMA) y el estoma descompresivo (ED). Las PMA se recomiendan en pacientes con síntomas obstructivos secundarios a CC izquierdo que tienen un riesgo elevado de mortalidad postoperatoria. Recientes estudios han revelado un incremento del uso del estoma descompresivo (ED) como puente a la cirugía siendo una alternativa a la resección de emergencia (RE) para el CC obstructivo del lado izquierdo. Cuando se comparan ambas técnicas, existe superioridad de las PMA sobre el ED en relación con menor riesgo de muerte, necesidad de estomas, costos, inicio más temprano de la quimioterapia.
\end{abstract}

Palabras clave: Prótesis metálica autoexpandible. Estoma. Cáncer de colon.

\section{Introducción}

El cáncer de colon (CC) representa el 10\% de todos los cánceres diagnosticados anualmente, constituye la segunda causa de muerte en hombres y mujeres por cáncer en el mundo1. Un número considerable de pacientes con CC se manifiestan clínicamente con datos de obstrucción colónica $(8-13 \%)^{2}$. Estos pacientes en su mayoría tienen edad avanzada, estado nutricional y clínico deficiente, distensión importante de asas intestinales. Esto ha permitido que se considere realizar terapia puente (prótesis metálica autoexpandible [PMA] o realización de estoma descompresivo [ED]) antes de la cirugía electiva ${ }^{3}$. La ASGE y ESGE recomiendan la utilización de PMA en pacientes con síntomas obstructivos secundarios a CC izquierdo que tienen un riesgo elevado de mortalidad postoperatoria (ASA $>3$ y edad $>70$ años) como una alternativa a la cirugía de emergencia ${ }^{2}$. Diversos estudios publicados (aleatorizados controlados, revisiones sistemáticas) coinciden que las
PMA son el tratamiento preferido para la paliación de la obstrucción maligna por CC, con tasas de éxito técnico del $88-100 \%$ y éxito clínico hasta en un $93 \%$, menor tasa de mortalidad comparado con la cirugía ( $4 \%$ vs. $11 \%$ ), menor estancia hospitalaria, tiempo más corto al inicio de la quimioterapia, menor necesidad de estomas ( $13 \%$ vs. $54 \%$ ). Las complicaciones a corto plazo son más frecuentes en el grupo de cirugía paliativa, mientras que las tardías en el grupo de PMA (perforación colónica $10 \%$, migración $(9 \%)$ y reobstrucción $(18 \%)^{2}$. Cuando se compara la colocación de PMA vs. realización de estomas, se observa una reducción importante en costo y mediana de días hospitalarios con significancia estadística para las PMA. No existe diferencia estadística en ambas terapias puente en relación con tasas de recurrencia locorregional a 3 años, tasa de supervivencia global a 3 años. Sin embargo, se requieren estudios aleatorizados controlados para validar estos datos ${ }^{3,4}$.

\section{Correspondencia:}


En la Semana de Enfermedades Digestivas 2020 (DDW) se aceptaron un total de 7 trabajos relacionados a paliación del cáncer de colon con PMA o ED.

Hajifathalian et al. evaluaron el efecto de la colocación de la prótesis metálica autoexpandible de colon (PMAC) en la mortalidad hospitalaria y la necesidad de cirugía. La colocación de la PMA se asoció con riesgo menor de muerte durante el ingreso (3.5\%, diferencia riesgo absoluto (DRA) $-3.5 \%$ [IC 95\%: -1.5 a $-5.6 \%$ ] $p=0.001)$ y una probabilidad menor de necesidad de cirugía durante el ingreso (29\%, DRA -29\% [IC 95\%: $-25 a-32 \%] p<0.001)$. No se observó diferencia en el tiempo de hospitalización, pero si a costo más elevado. Cuando se realizó el análisis univariado, se observó asociación significativa entre la colocación de la PMA y menor riesgo de muerte y cirugía en pacientes con CC izquierdo así como necesidad de creación de estoma (DRA -2.9\% [IC 95\%: -1.1 a 4.6\%] $p=0.001$, DRA $-32 \%$ [IC 95\%: $-29 \%$ a $-36 \%$ ] $p<0.001$ y DRA $-25 \%$ [IC 95\%: -33 a 17\%] $p<0.001$, respectivamente). La colocación de PMA en obstrucción en colon derecho no tuvo significancia en riesgo de muerte y creación de estoma, pero sí en la necesidad de cirugía $\mathrm{p}<0.001^{5}$.

En otro estudio retrospectivo realizado en Japón sobre la seguridad de las PMA en paliación de CC, la tasa de éxito clínico fue del $89 \%$ y éxito técnico $100 \%$. Hubo 2 casos de complicaciones en fase temprana (0-30 días-perforación de colon ascendente). Una complicación en fase tardía en 3 casos (obstrucción recurrente). EL tiempo promedio de reanudación de la alimentación oral fue de 2 días (0-4 días), duración promedio de la permeabilidad de la PMA fue de 148 días (35-1634 días). La paliación del CC con PMA es segura y efectiva en población de edad avanzada, con impacto positivo en esperanza y calidad de vida ${ }^{6}$.

Veld, et al. en un estudio nacional prospectivo compararon la estratificación de riesgo de la terapia puente antes de la cirugía vs. resección de emergencia con descompresión mediante estoma, en pacientes con obstrucción maligna por CC izquierdo. Se incluyeron un total de 2587 pacientes. En los pacientes con terapia puente (colocación de PMA) se observó significativamente menos estomas permanentes que en el grupo de resección de emergencia, con reducción de riesgo absoluto (RRA) que varía de $9.5 \%$ en pacientes de bajo riesgo (tumor sin invasión local, $<70$ años, ASA I y II) a $42.7 \%$ en pacientes de alto riesgo (tumor localmente avanzado, >70 años, ASA III y IV). No se observó diferencia significativa en la mortalidad a 90 días en ambos grupo ${ }^{7}$.
$\mathrm{Li}$, et al. en un estudio multicéntrico evaluaron los resultados de la colocación de PMA en CC obstructivo respecto a la recurrencia del cáncer y las tasas de ostomías. No hubo diferencia estadísticamente significativa en la tasa de recurrencia a 6 meses y a 1 año vs. pacientes que fueron sometidos a cirugía inicial por CC obstructivo o no obstructivo (3\%, $15 \%$ y $5 \%$ respectivamente, $p 0.156$ y 1.00). En términos de tasas de ostomía, el grupo de PMA tuvo una tasa de ostomía a los 6 meses del $23 \%$ y $16 \%$ a 1 año, en comparación con $41 \%$ y $33 \%$ respectivamente (valores p 0.065 y 0.066 ) en la cirugía inicial para CC obstructivo, y 32 y $16 \%$, respectivamente $(p=0.425$ y 1.00$)$ en el grupo de CC no obstructivo. Concluyendo que la colocación de PMA preoperatorio no se asoció con un mayor riesgo de recurrencia de cáncer hasta un año después de la cirugía ${ }^{8}$.

Bakheet, et al. desarrollaron una prótesis metálica autoexpandible recubierta con nanopartículas de oro (NO) con calentamiento local que induce supresión del crecimiento tumoral. La efectividad se investigó en un modelo de ratón ortotópico de cáncer de colon humano. Se dividieron de forma aleatoria en 3 grupos: Grupo A (PMA NO sin irradiación con láser), grupo B (PMA sin recubrimiento con irradiación con láser) y grupo $C$ (PMA NO con irradiación con láser). En el seguimiento por microendoscopia después de una semana se observó reducción del tamaño tumoral de manera significativa el grupo $C$ (media \pm DS $1.13 \pm .35$ ) en comparación con el grupo A $(2.7 \pm .49)$ y el grupo B $(2.6 \pm .53) ; p=<.001^{9}$.

En un estudio retrospectivo, se comparó el estoma descompresivo vs. la resección de emergencia. Se incluyeron 236 pacientes con ED y 472 con RE, mediana de seguimiento de 26 y 25.5 meses respectivamente $(p=0.064)$. En comparación a la RE, el ED resultó en más resecciones laparoscópicas (56.8\% versus $9.2 \%, p<0.001)$ y más anastomosis primarias (88.5\% versus $40.7 \%, p<0.001$ ). Pacientes con ED tenían un menor riesgo de mortalidad a los 90 días ( $1.7 \%$ versus $7.3 \% ; p=0.006)$ y una mejor supervivencia general a 3 años $(79.4 \%$ versus $73.3 \%$; HR 0.36 ; IC 95\%: 0.20-0.65; $p<0.001$ ), las tasas de estoma permanente $(18.8 \%$ versus $33.4 \%, p<0.001)$ fueron más bajas después del ED ${ }^{10}$.

\section{Conclusiones}

La evidencia actual disponible demuestra superioridad de las prótesis metálicas autoexpandible sobre los estomas de descompresión en cuanto a menor riesgo 
Endoscopia. 2020;32(Supl 1)

de muerte, necesidad de estomas, costos e inicio más temprano de la quimioterapia.

\section{Conflicto de intereses}

Los autores declaran no tener conflicto de intereses alguno.

\section{Financiamiento}

Los autores no recibieron patrocinio para llevar a cabo este artículo.

\section{Bibliografía}

1. Dekker E, Tanis $P$, Vleugels J, et al. Colorectal Cancer. The Lancet 2019;394(10207):1467-1580.
2. Van Hooft Jeanin E et al. Self-expandable metal stents for obstructing colonic and extracolonic cancer: European Society of Gastrointestinal Endoscopy (ESGE) Clinical Guideline. Endoscopy 2014; 46: 990-1002.

3. Veld J, Amelung F, Borstlap W, et al. Comparison of Decompressing Stoma vs Stent as a Bridge to Surgery for Left-Sided Obstructive Colon Cancer. JAMA Surg. 2020;155(3):206-215. doi:10.1001/jamasurg.2019.5466.

4. Sagar J. Role of colonic stents in the management of colorectal Cancers. World J Gastrointest Endosc 2016; 8(4): 198-204.

5. Hajifathalian K, Mehta A, Sampath K, et al. Colonic stent placement is associated with lower risk of in-hospital death and need for surgery among patients admitted with malignant large bowel obstruction: an analysis of a large national sample. Presentation Mo1742 - 2020. DDW 2020.

6. Mori C. Nishino T, et al. Usefullness of colonic stenting for palliative treatment of malignant colonic obstruction. Presentation mo1704 - 2020 asge.

7. Veld J, Amelung F, et al. Risk-stratified comparison of bridge to surgery approaches versus emergency resection in patients with left-sided obstructive colon cancer: a nationwide study. Presentation 897. DDW 2020.

8. Li X, Tintara S, et al. Preoperative colonic stenting is not associated with an increased risk of recurrent cancer compared to upfront surgery in patients with obstructive colorectal cancer, a multicenter study. Mo1623. DDW 2020.

9. Bakheet $\mathrm{N}$, Tao $\mathrm{H}$, et al. Local photothermal therapy using nano functionalized self expandable metal stent in an orthotopic mouse model of human colon cancer. Presentation Mo1688. DDW 2020.

10. Veld J, Amelung F, et al. Decompressing stoma versus emergency resection for left-sided obstructive colon cancer: a nationwide, propensity score matched study. Presentation 177. DDW 2020. 